DOI:10.1145/2908732

\title{
The IANA Transition
}

\section{This month I would like to explore the notable proposal sent in March 2016 by the Internet Corporation for Assigned Names and Numbers (ICANN) to the U.S.}

Department of Commerce, National Telecommunication and Information Agency (NTIA) to end the long-standing contractual relationship between ICANN and NTIA for the conduct of the Internet Assigned Numbers Authority functions ("IANA functions"). ICANN was formed in the fall of 1998 in response to a White House "White Paper" issued by Ira Magaziner, then a senior advisor for policy to President Bill Clinton. ICANN would undertake to form a private sector entity to carry out the coordinated assignment of Internet domain names, Internet addresses, and the maintenance of parameter registries needed for the operation of the suite of protocols used in the Internet.

These functions had been managed by Jonathan Postel acting as the IANA at USC Information Sciences Institute (and other earlier institutions where Postel had worked) under various government contracts. By 1996, the Internet was experiencing its so-called "dot boom" and the potential scale and liabilities of carrying out the IANA functions led to a serious effort to institutionalize the operation. For lack of space, I will leave out two years of community debate and fast-forward to the creation of ICANN to fulfill these functions. ICANN was conceived as a multistakeholder organization drawing on input from the private sector, civil society, governments of the world, and the technical community for the development of policy for the IANA functions and for the coordination of the multiple parties having a role in managing these unique identifiers and parameters.
In 1998, many organizations were involved in the evolution and operation of the Internet, its Domain Name System (DNS), Internet address allocation, and standards development. The Internet Society, founded in 1991, housed the standards-oriented Internet Architecture Board and the Internet Engineering Task Force. There were then three Regional Internet Registries (RIRs) for Internet address allocation-RIPE-NCC, APNIC, and ARINand two more to follow later (LACNIC and AFRINIC). There were nominally 13 DNS Root Server operators providing top-level domain name resolution. Verisign generated and distributed the official domain name root zone based on input from IANA and, under the terms of the NTIA/ICANN contract, authorization from NTIA. Many domain name registries and registrars were created to support DNS operation.

The original plan was for ICANN to operate under NTIA oversight for a few years and then operate as an independent organization. In fact, the contractual obligations extended from 1998 to the present. In March 2014, however, NTIA proposed this contractual relationship for the IANA functions should be ended and ICANN be allowed to perform the IANA functions independently. In March 2016, ICANN delivered to NTIA its consolidated proposal from all the constituent parties for the transition from the present contractual relationship to independent op eration. The two-year effort leading to this comprehensive proposal was not

\section{Vinton G. Cert}

without considerable debate among all the parties. Many ideas were surfaced, analyzed, argued over, adopted, adapted, or discarded leading to a consolidated result. The Department of Commerce and the U.S. Congress will be evaluating the proposed new modus operandi in the weeks ahead.

Some fears have been voiced that the complex proposal poses risks that authoritarian governments within the ICANN Governmental Advisory Committee (GAC) or through some external means might wrest control of ICANN from its multi-stakeholder constituencies. While the proposal should be evaluated on all its merits, I am persuaded the terms and conditions of the proposed operating practices are well protected against such an outcome. A great many conditions must be satisfied before the more extraordinary powers of the sole designator can be exercised. The headquarters of ICANN will remain in the U.S. The many entities that cooperate with ICANN to manage core Internet identifier administration have expressed full support for the proposal.

If I have any trepidation about the proposal it is associated with its general complexity. As the former chairman of ICANN, I am no stranger to the evolution of ICANN's structure and processes and their relative intricacy. The new proposal adds its own unique aspects to this tendency and it remains to be seen how well the system will work. However, ICANN has shown a remarkable ability to reform and adapt at need and I believe that capacity is preserved under the new proposal. There is still a good deal of work ahead to actually implement what is ultimately approved, but I feel confident this community is capable of achieving a successful outcome. c

Vinton G. Cerf is vice president and Chief Internet Evangelist at Google. He served as ACM president from 2012-2014.

Copyright held by author 